\title{
Metal-Dielectric Transition in Hydrogen
}

\author{
V.T. Shvets* And A.S. Vlasenko \\ Odessa State Academy of Refrigeration \\ Dvoryanskaya 1/3, Odessa, 65082, Ukraine
}

(Received April 1, 2008)

\begin{abstract}
The electrical resistivity of liquid metallic hydrogen at a temperature of $3000 \mathrm{~K}$ and a density of $0.35 \mathrm{~mol} / \mathrm{cm}^{3}$ is calculated. Hydrogen is considered as a three-component system consisting of electrons, protons, and neutral hydrogen atoms. The second order of perturbation theory in electron-proton and electron-atom interactions is used to determine the inverse relaxation time for electric conductivity. The Coulomb electron-electron interaction is taken into account in the random phase approximation and the exchange interaction and correlation of conductivity electrons are included in the local-field approximation. The model of hard spheres is used for the proton and atomic subsystems. The concentration of the electrically neutral atomic component proved to be significantly lower than the value assumed by the discoverers of metallic hydrogen.
\end{abstract}

PACS numbers: 72.10.-d, 72.15.-v, 72.15.Cz, 72.15.Lh

\section{Introduction}

The existence of hydrogen in the metallic state was theoretically predicted in 1935 [1]. A number of studies devoted to the theoretical description of its properties were published in the subsequent years (see, e.g., $[2,3]$ ). A qualitative breakthrough in the studies of metallic hydrogen occurred after a number of experiments on shock compression of both molecular hydrogen in the liquid state and hydrogen and deuterium plasma. Those experiments showed that electric conductivity increased steeply for certain combinations of density, pressure, and temperature [4-9]. Specific values of these parameters depended on the initial state of hydrogen (i.e., the state before compression). The values quoted by different experimentalist teams also somewhat differed. To make the quantitative calculations performed in this study more certain, we use the values of these parameters obtained, e.g., in [4]. However, since the experimental studies of [5] are fairly comprehensive, we use some experimental results of this study for interpreting the theoretical results

*corresponding author; e-mail: valtar@paco.net 
that we obtained. According to [4], molecular hydrogen in the liquid state was subjected to shock compression up to the pressures in the range 0.93-1.80 Mbar at the temperatures $2200-4400 \mathrm{~K}$ and densities $0.28-0.36 \mathrm{~mol} / \mathrm{cm}^{3}$. If pressure was increased to $1.4 \mathrm{Mbar}$, temperature to $3000 \mathrm{~K}$, and density to $0.32 \mathrm{~mol} / \mathrm{cm}^{3}$, the width of the band gap reduced to the specimen temperature $(3000 \mathrm{~K})$. This point was considered by the authors of [4] as the point of the transition of molecular hydrogen into the metallic state, although hydrogen in this state is still a semiconductor. If the hydrogen density was increased further, the band gap vanished rapidly [4] and its presence could be neglected at a density of $0.35 \mathrm{~mol} / \mathrm{cm}^{3}$. The electrical resistivity for the hydrogen transition to the metallic state amounted to the values $500 \mu \Omega \mathrm{cm}$ in [4] and $<1000 \mu \Omega \mathrm{cm}$ in [6] that are specific to heated metals.

According to the results of many experiments and primarily those discussed above, the existence of hydrogen in solid state may be considered to be a reliably established experimental phenomenon. This possibility is theoretically confirmed by quantum-mechanical calculations made by the Monte Carlo method [10]. After the impressive breakthrough in the experimental studies of hydrogen in the metallic state, theoretical studies of its various properties in broad ranges of densities and pressures [11, 12] become increasingly important. This refers, in particular, to the conditions that are specific to the cores of giant planets in the solar system, namely, Jupiter, Saturn, Uranus, and Neptune. The theoretical studies may be strongly encouraged by the fact that the methods for shock compression of the matter that are used in laboratory conditions allow experimentalists to obtain virtually any pressures up to a gigabar range [12].

The aim of this study is to analyze the possibility of using the model of almost free electrons for describing the electrical resistivity of metallic hydrogen in quantitative terms and determining the metallization degree of hydrogen in the metal-dielectric transition. It should be noted that the degree of hydrogen metallization is also of importance for analyzing the equilibrium properties of metallic hydrogen [13].

The currently existing methods for calculating electrical resistivity, which use both the Ziman formula [3, 14] and computer simulation [15], are based on the assumption that metallic hydrogen is a two-component system that consists of only protons and electrons. In both cases, the values of the electrical resistivity proved to be approximately $20 \mu \Omega \mathrm{cm}$.

The inclusion of higher-order terms of perturbation theory in electron-ion interaction is important in considering the electronic transport phenomena in disordered metals. These terms are fairly significant in the entire range where the metallic phase exists and are quite well studied in simple liquid metals [16-25]. Studies of these terms also began for metallic hydrogen in broad ranges of temperatures and densities [26]. If the higher-order terms of perturbation theory are taken into account, the electrical resistivity of metallic hydrogen calculated under the 
assumption that hydrogen atoms are fully ionized at a density of $0.35 \mathrm{~mol} / \mathrm{cm}^{3}$ and a temperature of $3000 \mathrm{~K}$, which correspond to the conditions of the experiments in which metallic hydrogen was produced, proves to be approximately $50 \mu \Omega \mathrm{cm}$. However, this electrical resistivity is still an order of magnitude smaller than its experimental value. The authors of [4] were the first to notice this discrepancy between the experimental and theoretical values of electrical resistivity. They assumed that the presence of neutral hydrogen atoms or molecules was the main factor that explained the observed discrepancy. In their opinion, the fraction of protons, i.e., ionized hydrogen atoms, should be about $5 \%$ of the mass of the system. However, the electrical resistivity of metallic hydrogen as a complex multicomponent system has not been calculated theoretically. An aim of this study is to fill this gap.

\section{Metal-dielectric transition}

For simple disordered metals with relatively high conductivity, the electrical resistivity $R$ is determined in the relaxation time approximation as follows:

$$
R=\frac{m}{e^{2} n} \frac{1}{\tau}
$$

where $n$ is the density of electron gas, $T$ is the relaxation time for the electric conduction process, $e$ is the elementary charge, and $m$ is the electron mass.

In the second order of perturbation theory in electron-ion interaction, the following expression may be derived in the high-temperature limit for the inverse relaxation time (the Ziman formula) [14, 16-26]:

$$
\tau^{-1}=\frac{m}{12 \pi^{3} h^{3}} \int_{0}^{2 k_{F}} W^{2}(q) S(q) q^{3} \mathrm{~d} q .
$$

Here, $S(q)$ is a pair static structure factor of the ion subsystem and $W(q)$ is the screened potential of electron-ion interaction. For metallic hydrogen, the potential of electron-proton interaction is $W_{\mathrm{p}}(q)=-V(q) / e(q)$, where $V(q)=4 n e^{2} / q^{2}$ is the Fourier transform of the potential of the Coulomb proton-proton or electronproton interaction, $\varepsilon(q)=1+[V(q)+V(q)] \pi_{0} q$ is the effective relative permittivity of the electron gas in the random phase approximation, $V(q)=\frac{-2 \pi e^{2}}{q^{2}+\lambda k_{F}^{2}}$ is the potential of exchange interaction and electron-gas correlations $\lambda \approx 2[27], k_{F}$ is the Fermi wave vector, and $\pi_{0}(q)$ is the polarization function of the free electron gas.

Metallic hydrogen is the only system for which the unscreened potential of the electron-proton interaction is accurately known. However, the electron-atom interaction is very complex [28]. It should be noted that, for the interparticle distances specific to metals, electrostatic interaction is the only significant component of this interaction and we take into account only this component.

We assume that, when metallic hydrogen is produced, the molecular phase of liquid hydrogen transforms into the atomic phase [9] and only some atoms are later ionized. 
The transition to the electrical resistivity of a binary alloy or solution of a metal in a nonconducting liquid may be formally performed with the following substitution [29]:

$$
W^{2}(q) S(q) \rightarrow c_{1} c_{2}\left[W_{1}(q)-W_{2}(q)\right]^{2}+\sum_{i, j=1}^{2} c_{i} c_{j} W_{i}(q) W_{j}(q) S_{i j}(q)
$$

Here $c_{1}=c_{\mathrm{a}}$ and $c_{2}=c_{\mathrm{p}}$ are the concentrations of hydrogen atoms and protons, respectively; $S_{11}(q)=S_{\mathrm{a}}(q)$ is the structure factor of the atomic subsystem; $S_{22}(q)=S_{\mathrm{p}}(q)$ is the structure factor of the proton subsystem; $S_{12}(q)=S_{21}(q)=S_{\text {ap }}(q)$ are the mixed structure factors of atoms and protons; $W_{1}(q)=W_{\mathrm{a}}(q) ; W_{2}(q)=W_{\mathrm{p}}(q)$; and $W_{\mathrm{a}}(q)=\frac{-U(q)}{\varepsilon(q)}$ is the screened potential of electron-atom interaction, where $U(q)=\frac{4 \pi e^{2}}{q^{2}}\left[1-\frac{16}{\left(4+q^{2}\right)^{2}}\right]$ is the Fourier transform of the electrostatic interactions between the electron and hydrogen atom [30].

We determine the diameter of hard spheres (or the minimal distance to which protons may approach at a given temperature) from the condition that the kinetic and potential energies of protons are equal when the distance between them is minimal $[26,31]$. The diameter of the hard spheres found in this way is a function of the density and temperature. As a result, all the quantities that depend on the diameter of the hard spheres also depend on the density and temperature.

Subsequent calculations may be significantly simplified without loss of accuracy if the Bohr radius is taken as the radius of the hard sphere that corresponds to the hydrogen atom. Indeed, the temperature and density dependence of the diameter of the hard spheres that correspond to atoms is determined by only the screening effect of the electron subsystem. This effect is fairly small for the short-range atomic potential.

The density and temperature of the system are natural external parameters. The presence of internal parameters in the system is related to the model character of calculations. Since the form factor of electron-proton interaction is accurately known, the adopted approximation for the form factor of electron-atom interaction does not contain fitting parameters, and since the electron subsystem is described in the random phase approximation, only the proton and atomic subsystems may be sources of the internal parameters of the system. If the exact solution of the Percus-Yevick equation for the model of hard spheres is used for the partial pair structure factors of the proton and atomic subsystems, the parameters associated with these subsystems actually appear in the theory (diameters of hard spheres $\sigma_{\mathrm{a}}$ and $\sigma_{\mathrm{p}}$ and the parameters of packing density $\eta_{\mathrm{a}}$ and $\eta_{\mathrm{p}}$, which are interrelated by simple formulae). One can easily check that only one of these parameters is actually independent. It is convenient to adopt the diameter of the hard spheres that correspond to protons as this independent parameter. If the temperature of the system is also known, the remaining parameter of the system may also be determined as it was described above. 


\section{Results and discussion}

Figure 1 shows the electrical resistivity of metallic hydrogen in the second order of perturbation theory in the potential of electron-proton and electronatom interactions as a function of the concentration of protons (electrons) or hydrogen atoms. The density and temperature of this three-component system correspond to the conditions of the experiments in which metallic hydrogen was produced [4]. The proton concentration varies from the value that is close to the possible maximum, for which electrical resistivity is approximately $20 \mu \Omega \mathrm{cm}$, and to the minimum when electrical resistivity attains the measured value $500 \mu \Omega \mathrm{cm}$ [4]. This minimal concentration of protons is of interest for us. According to our calculations, this minimum is $10 \%$. For comparison, the same figure shows the density dependence of the electrical resistivity of metallic hydrogen determined under the assumption that all the electrons in metallic hydrogen are collectivized. It is natural then to use the Ziman formula for the electrical resistivity of pure metals in which the presence of neutral hydrogen atoms is neglected. In this case, the experimental value of the electrical resistivity is reproduced for the density of protons that makes $7 \%$ of the specimen density. This value almost coincides with the estimate (5\%) presented in [4]. This estimate seems also to be based on the analysis of the Ziman formula. One more conclusion is that the role of electron scattering from neutral atoms is fairly significant as compared to their scattering from protons.

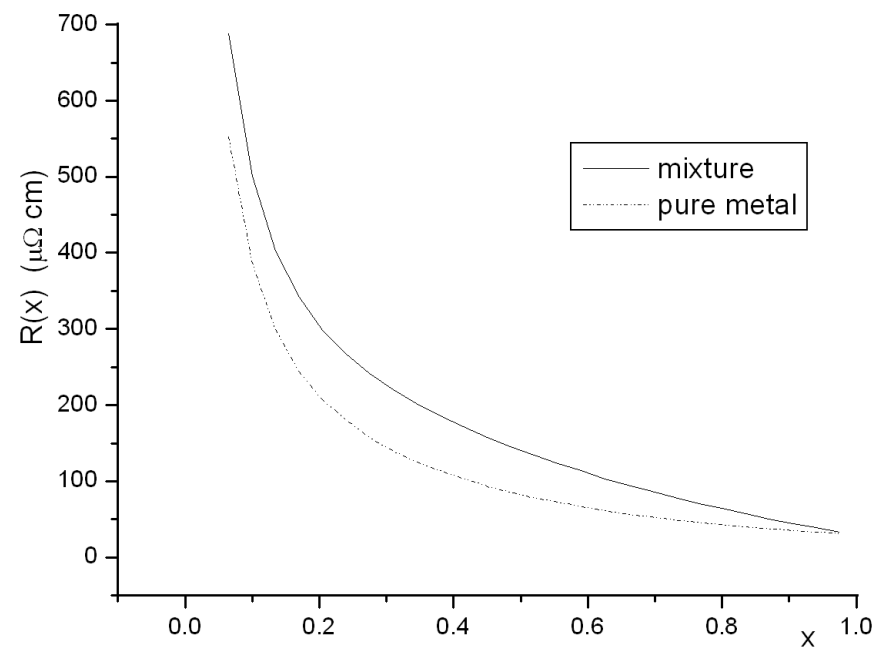

Fig. 1. Electrical resistivity of metallic hydrogen at $T=3000 \mathrm{~K}$ vs. the density of the proton subsystem.

An apparent specific feature of the contribution from scattering on hydrogen atoms is that it tends to zero not only when the concentration of hydrogen atoms approaches zero, but also when this concentration becomes maximally high. The 
latter phenomenon is attributed to the concurrent decrease in the density of conduction electrons so that the behavior of the electron-atom and electron-proton form factors for only small values of the wave vector becomes significant in integral (2). The screened form factor of electron-proton interaction tends to its maximum possible value and the form factor of electron-atom interaction tends to zero.

In our opinion, both estimates of the fraction of hydrogen atoms should be refined because, according to the Ioffe-Regel criterion [32], the model of almost free electrons fails if the mean free path of the conduction electrons approaches the distance between protons and the resistivity of the system approaches $200 \mu \Omega \mathrm{cm}$. One can determine the behavior of each specific metallic system where the IoffeRegel criterion holds only provided that higher orders of perturbation theory in electron-proton interaction are taken into account [16-26]. Since we only aim at estimating the order of magnitude of the effect, the electron scattering from neutral hydrogen atoms is neglected.

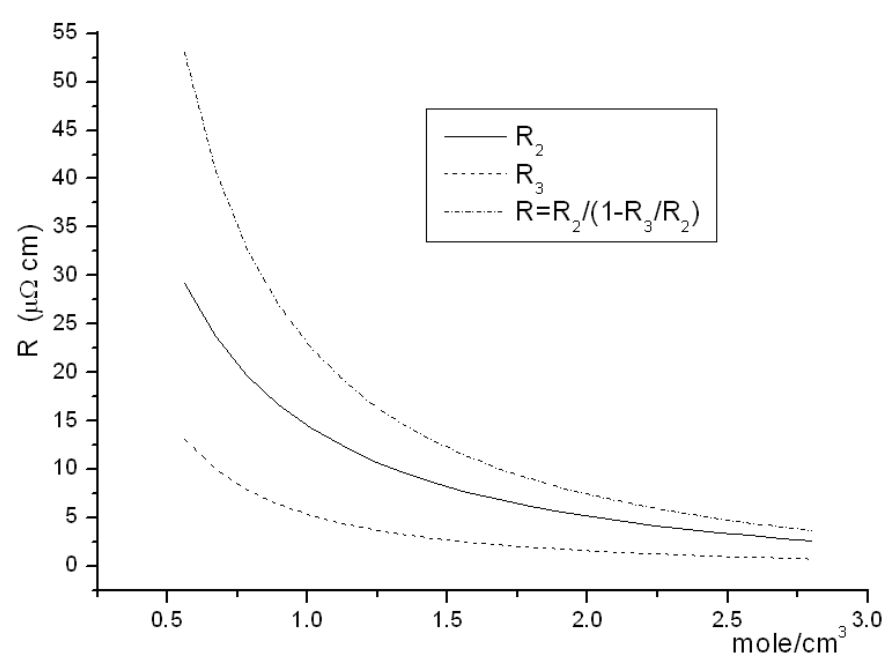

Fig. 2. Density dependence of the electrical resistivity of metallic hydrogen at $T=$ $3000 \mathrm{~K}$ calculated with the inclusion of higher-order terms of perturbation theory.

Figure 2 shows the contributions from the second $\left(R_{2}\right)$ and the third $\left(R_{3}\right)$ orders in electron-proton interaction to the electrical resistivity of metallic hydrogen as a function of the density of the proton subsystem calculated according to [26]. One can see that the third-order contribution is comparable to the second-order contribution for all the studied densities. It is especially large at relatively low densities. This figure also shows the result of the approximate summation of the perturbation-theory series $R=\frac{R_{2}}{1-R_{3} / R_{2}}$. It is seen that the series converges even for a resistivity of $500 \mu \Omega \mathrm{cm}$. If the density of the system decreases further, the theoretical value of the resistivity obtained in the model of almost free electrons tends to infinity. 
The plot shown in Fig. 2 may be used for determining the fraction of protons and neutral atoms under the conditions of the experiment in which hydrogen in the metallic state is obtained. One can see that the inclusion of the higher-order terms of perturbation theory shifts the lower estimate for the density of protons from $5-10 \%$ to $30-35 \%$. If the additional scattering of conduction electrons from hydrogen atoms is taken into account in the higher orders of perturbation theory, the electrical resistivity attains the experimental value at even higher densities of the proton subsystem. This theoretical conclusion agrees well with the experimental results of [5], where the fraction of ionized atoms was $\leq 40 \%$.

Thus, (i) the model of almost free electrons provides not only a qualitative but also a quantitative description of the electrical resistivity of metallic hydrogen; (ii) metallic hydrogen at a temperature of $3000 \mathrm{~K}$ and a density of $0.35 \mathrm{~mol} / \mathrm{cm}^{3}$ contains, most probably, no less than three components. The fraction of the proton subsystem in the total density of the metal is no less than $30-35 \%$ rather than $5 \%$ as was concluded by the authors of [4]. This value fully agrees with the experimental results reported in [5].

\section{References}

[1] E. Wigner, H.B. Huntington, J. Chem. Phys. 3, 764 (1935).

[2] E.G. Brovman, Yu. Kagan, A. Holas, Zh. Eksp. Teor. Fiz. 61, 2429 (1971) [Sov. Phys. JETP 34, 1300 (1971)].

[3] D.J. Stevenson, N.W. Ashcroft, Phys. Rev. A 9, 782 (1974).

[4] S.T. Weir, A.C. Mitchell, W.J. Nellis, Phys. Rev. Lett. 76, 1860 (1996).

[5] V.E. Fortov, V.Ya. Ternovoi, S.V. Kvitov, Pis'ma Zh. Eksp. Teor. Fiz. 69, 874 (1999) [JETP Lett. 69, 926 (1999)].

6] V.Ya. Ternovoi, A.S. Filimonov, V.E. Fortov, Physica B (Amsterdam) 265, 6 (1999).

[7] V.E. Fortov, V.Ya. Ternovoi, M.V. Zhernokletov, Zh. Eksp. Teor. Fiz. 124, 288 (2003) [JETP 97, 259 (2003)].

[8] S.K. Grishechkin, S.K. Gruzdev, V.K. Gryaznov, Pis'ma Zh. Eksp. Teor. Fiz. 80, 452 (2004) [JETP Lett. 80, 398 (2004)].

[9] W.J. Nellis, Rep. Prog. Phys. 69, 1479 (2006).

[10] V.S. Filinov, V.E. Fortov, M. Bonitz, P.R. Levashov, Pis'ma Zh. Eksp. Teor. Fiz. 74, 422 (2001) [JETP Lett. 74, 384 (2001)].

[11] E.G. Maksimov, Yu.I. Shilov, Usp. Fiz. Nauk 169, 1223 (1999) [Phys. Usp. 42, 1121 (1999)].

[12] V.E. Fortov, Usp. Fiz. Nauk 177, 347 (2007).

[13] V.V. Kechin, Pis'ma Zh. Eksp. Teor. Fiz. 79, 46 (2004) [JETP Lett. 79, 40 (2004)].

[14] J.M. Ziman, Philos. Mag. 6, 1013 (1961).

[15] I. Kwon, L. Collins, J. Kress, N. Troullier, Phys. Rev. E 54, 2844 (1996).

[16] B. Springer, Phys. Rev. 136, 115 (1964). 
[17] J. Rubio, J. Phys. C 2, 288 (1969).

[18] T. Neal, Phys. Fluid 13, 249 (1970).

[19] N.W. Ashcroft, W. Schaich, Phys. Rev. B 1, 1370 (1970).

[20] A. Bringer, D. Wagner, Z. Phys. 241, 295 (1971).

[21] J. Popielawski, Physica (Amsterdam) 78, 97 (1974).

[22] J. Gorecki, J. Popielawski, J. Phys. F 13, 2107 (1983).

[23] V.T. Shvets, E.V. Belov, Acta Phys. Pol. A 96, 741 (1999).

[24] V.T. Shvets, Green's Function Methods in the Theory of Metals, Latstar, Odessa 2002 (in Russian).

[25] V.T. Shvets, Physics of Disordered Metals Mayak, Odessa 2002 (in Russian).

[26] V.T. Shvets, S.V. Savenko, Ye.K. Malynovski, Condens. Matter Phys. 9, 127 (2006).

[27] D.J.M. Geldart, S.H. Vosko, Can. J. Phys. 44, 2137 (1966).

[28] L.M. Biberman, V.S. Vorob'ev, I.T. Yakubov, Kinetics of Nonequilibrium Low-Temperature Plasmas, Nauka, Moscow 1982; Consultants Bureau, New York 1987.

[29] T.E. Faber, J.M. Ziman, Philos. Mag. 11, 153 (1965).

[30] V.G. Levich, Yu. A. Vdovin, V.A. Myamlin, Course of Theoretical Physics, Nauka, Moscow 1971, Vol. 2 (in Russian).

[31] V.T. Shvets, Zh. Eksp. Teor. Fiz. 131, 743 (2007) [JETP 104, 655 (2007)].

[32] A.F. Ioffe, A.R. Regel, Prog. Semicond. 4, 237 (1960). 